\title{
Integration and Evolution of Data Mining Models in Ubiquitous Health Telemonitoring Systems
}

\author{
Vladimer Kobayashi, Pierre Maret, \\ Fabrice Muhlenbach, and Pierre-René Lhérisson \\ Université de Lyon, CNRS, UMR 5516, Laboratoire Hubert Curien \\ 42000 Saint-Etienne, France \\ Vladimer.Kobayashi, Pierre.Maret, Fabrice.Muhlenbach@univ-st-etienne.fr
}

\begin{abstract}
Ubiquitous Health Telemonitoring Systems collect low level data with the aim to ameliorate the health condition of patients. Models from data mining are created to compute indicators regarding their status and activity (habits, abnormalities). Models can also help generate feedbacks and recommendations for patients as well as for remote formal and informal care givers. Essential features are that the models can be easily updated whenever new information is available and that data generated from the models can be readily accessible as well as sensed data. This paper addresses the challenge of conveniently incorporating in a Ubiquitous Health Telemonitoring System the creation, the use, and the updating of data mining models. We conducted first runs and generated results showing the feasibility as well as the effectiveness of the system.
\end{abstract}

Key words: Ubiquitous systems, telemonitoring, eHealth, data mining models

\section{Introduction}

Healthcare provides a rich domain for the application of ubiquitous computing [3. We used the term Ubiquitous Health Telemonitoring System (UHTS) to encapsulate the conglomeration of various technologies and techniques that facilitate human health monitoring at a distance. Technologies and techniques inconspicuously work together to give patients freedom in mobility, thereby maintaining their independent living. A quick survey of literature would reveal that a significant number of UHTSs have already been implemented. At their core, health telemonitoring systems are expected to accomplish two basic tasks, i.e. able to continuously collect data from patients wherever they are, and allow communication between patient and medical staff at a distance.

We can distinguish existing UHTSs as to how they handle and process the data they collect. It is worth noting that many systems only use, if they use at all, low level data processing techniques. Data sensed from patients are transmitted and screened and selected data are stored in static and centralized repositories and sporadically accessed by the concerned individuals. There are efforts to 
incorporate higher level analytics to the stored data 4 but there is no straightforward solution as to how the results in the form of knowledge models (obtained from applying higher level analytics such as data mining techniques) are integrated back to the system and how these knowledge models could be evolved in the presence of new data. In retrospect existing systems lack an important layer that is altogether able to develop, incorporate, and evolve models as well as make the results accessible to various stakeholders in a manner that is relevant, timely, and unrestricted by location. Our belief is that any system that collects data must also make use of the data to improve the operations of the system and subsequently would lead to the amelioration of the condition of the main target user. In the case of UHTS the main users are the patients to which we aim to improve or maintain their health condition. The motivation is to create models that can compute indicators regarding the status and activity of patients. Moreover the indicators constitute different levels of information (from detailed to coarse-grained) that will also be used either to generate automatic suited feedbacks (to patients or to care givers). An essential feature is that the models can be conveniently updated whenever new information or new scenarios are available. The challenge is for the entire process of creating, using, and updating models should be efficiently incorporated in the ubiquitous telemonitoring system and, to generate information available to the right stakeholders. In this paper we offered a solution to the challenge through the development of an additional component that permitted the associated tasks.

\section{Features of Ubiquitous Health Telemonitoring System}

The primary users of a UHTS are the patient and the medical practitioner, usually a physician. We can include more users as we add tasks and features in the system. The features of UHTS are enumerated next.

1. Collection, Transmission, and Storing of health data from patient.

2. Communication between patient and physician.

3. Communication with other health stakeholders forming the patient social support groups.

4. Access privileges mechanism to the health data, under the control of the patient and with the advice of the doctor

Additionally, we propose to consider these features:

5. Integration of models to generate higher level information from data.

6. Storing of the higher level information as health data.

7. Automatic recommendations and feedback to stakeholders (patient, etc.). 


\section{Requirements for Knowledge-Enriched Ubiquitous Health Telemonitoring System}

Our implementation of UHTS has been reported recently in a paper explaining its different components [2]. The system is aptly called CHISel'd (Comprehensive Health Information System). We enhanced this system by supplying a component that accommodated models from data mining procedures. Moreover it has the capability to generate and store higher-level information inferred from sensed data. We call it the Knowledge Engine (KE) component. We named the improved system: Knowledge-Enriched Ubiquitous Health Telemonitoring System (KEUHTS). Figure 1 shows the relationship among the different parts of a KEUHTS.

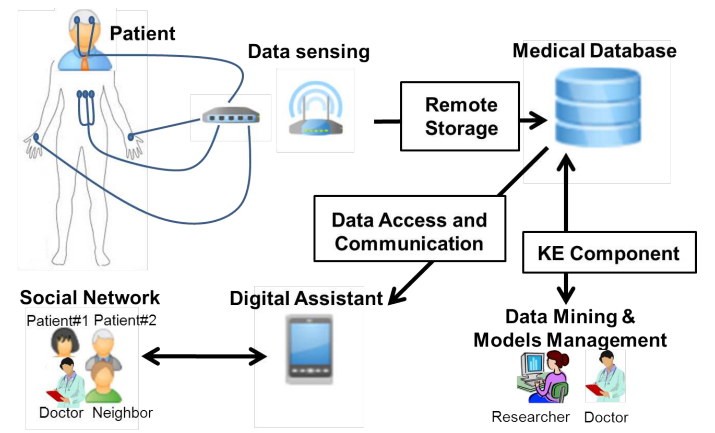

Fig. 1: The Knowledge-Enriched UHTS.

We identified four requirements of the Knowledge-Engine component:

1. Models from data mining could be easily added, modified, and deleted.

2. Possibility of patient-model customization within the platform.

3. Model-generated outputs (representing high level medical or activity information) are stored into the database.

4. Automatic recommendations and feedbacks are generated (through longterm use of the system) [1].

The intended users of the Knowledge-Engine component are the doctor and the researcher. The task of the researcher is to implement data mining algorithms and consequently to create the models. The role of the doctor is two-fold. The first role is to assist the researcher in the model creation process by providing domain expert knowledge and the second role is to validate the models. Also, the doctor is responsible for the assignment of feedbacks associated to the output of the models. 


\section{Implementation of the Knowledge Engine Component}

Here we explain an implementation of KE Component with respect to CHISel'd. We started by making a choice regarding the tools we used to create the engine.

We selected the $\mathrm{R}$ software ${ }^{1}$ to implement the data mining algorithms and we produced $\mathrm{R}$ scripts to store the created models. We picked the Java programming language to create an application that acted as the interface. The whole application is actually a combination of a Java interface (serving as the front-end) and the $\mathrm{R}$ software (serving as the back-end and doing all the model building and computational tasks). We call this application the P-JR (ProgramJava-R) application (Figure 2). What the doctor sees most of the time is the Java interface where majority of the activities such as model-patient assignment and process initiation/termination are effected. The Java interface also maintains a list of available patients and available models so that the doctor can easily make these associations.

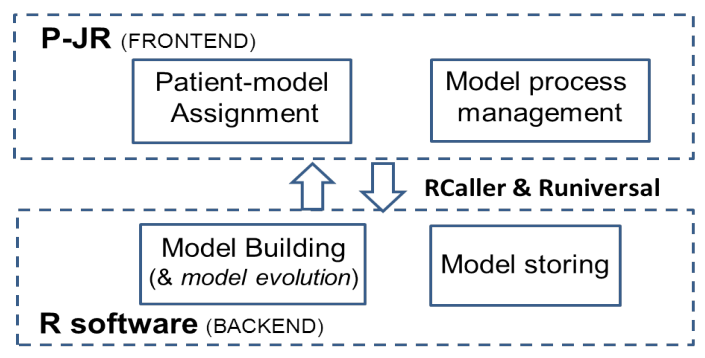

Fig. 2: The Knowledge-Engine showing the front-end and back-end programs.

In the P-JR application, when model running is activated it connects to the medical database to fetch the relevant raw data, when the data are collected the calculation commences, and finally the output from the model are written back to the database. In the database, we distinguished between raw data: the data collected directly from the patients; and context data: the data generated by the models. As for with raw data, the context data can also be accessed by stakeholders given the appropriate privileges.

We tested the KE component by creating test models and we have successfully completed the entire process. It starts by (a) entering the model (the doctor loads a R file and gives a description), then (b) the doctor assigns the model to a patient and activates the running of the model, next (c) each running of a model for a patient is a thread that can be stopped or killed, (d) the model reads raw data and generates output (context data) which is stored in the database, then (e) the data is made available for visualization on digital assistants, and (f) the raw and context data is used to generate new models or to modify existing ones and the steps are repeated.

\footnotetext{
1 http://www.r-project.org/
} 
Medical raw data as well as context data in the database can be accessed by stakeholders from their digital assistant (android application on a mobile device) and depending on their access rights ([2]). Through long-term use of the system we will be able to accumulate historical records regarding patient-model assignments and model-feedback associations. The gathered information will be used to improve the system to enable it to give automatic recommendations and oriented feedbacks.

\section{Conclusion and Future Work}

In this paper we have discussed and successfully demonstrated the development of a Knowledge Engine (KE) component that is crucial to any Ubiquitous Health Telemonitoring Systems (UHTS). The KE component is very well adapted for ubiquitous telemonitoring where raw data should be processed in real-time and high level information are computed and accessible on demand. The KE component is useful to implement the whole process, from model design to its integration and use into the system, i.e. the generation of context data. This is intended to help better monitor and support patients. Our approach makes it possible the dynamic inclusion of models created from data mining algorithms. We implemented the engine as a new component in an existing UHTS called CHISel'd which makes it a fully functional KE-UHTS. New data generated from the models is available in real-time and accessible from anywhere for instance on mobile devices. Also, new data is used to develop more models or to improve existing ones. Our next goal is to populate the model repository, conduct experiments to assess the over-all efficiency and reliability of the system, and to improve the operations in the interface.

\section{References}

1. Xavier Amatriain, Alejandro Jaimes, Nuria Oliver, and Josep Pujol. Data mining methods for recommender systems. In F. Ricci, L. Rokach, B. Shapira, and P. B. Kantor, editors, Recommender Systems Handbook, pages 39-71. Springer, 2011.

2. Juri Luca De Coi, Gwendal Delaunay, Adrien Martins Albino, Fabrice Muhlenbach, Pierre Maret, Guillaume Lopez, and Ichiro Yamada. The comprehensive health information system: a platform for privacy-aware and social health monitoring. In Proceedings of IADIS e-Health 2012. 2012.

3. YungBok Kim, Sun Yoo, and Daeyoung Kim. Ubiquitous healthcare: Technology and service. In N. Ichalkaranje, A. Ichalkaranje, and L.C. Jain, editors, Intelligent Paradigms for Assistive and Preventive Healthcare, volume 19 of Studies in Computational Intelligence, pages 1-35. Springer Berlin Heidelberg, 2006.

4. Viswanathan, Whangbo, and Yang. Data mining in ubiquitous healthcare. In Kimito Funatsu, editor, New Fundamental Technologies in Data Mining, pages 193-200. InTech, 2011. 